\title{
Cholesterol modification of Hedgehog family proteins
}

\author{
Juhee Jeong and Andrew P. McMahon \\ Department of Molecular and Cellular Biology, The Biolabs, Harvard University, Cambridge, Massachusetts, USA \\ J. Clin. Invest. 110:591-596 (2002). doi:10.1172/JCI200216506.
}

The Hedgehog $(H b)$ gene family encodes a group of secreted signaling molecules that are essential for growth and patterning of many different body parts of vertebrate and invertebrate embryos (1). Depending on the context, Hh signals can promote cell proliferation, prevent apoptosis, or induce specific cell fates. Hh family members can exert their effects not only on cells neighboring the source of the signal, but also over considerable distances (up to 30 -cell diameters), acting in at least some cases as classic morphogens. Such morphogens are signaling molecules that diffuse from a source to form a concentration gradient over an extended area of the target field and elicit different responses from cells according to their position within the gradient, which in turn reflects the dosage of the ligand they are exposed to.

In the fruit fly Drosophila, Hh patterns the embryonic ectoderm via short-range interactions with other signaling molecules (2). On the other hand, it employs two different strategies in larval wing imaginal discs: it induces a secondary signal (Decapentaplegic [Dpp]) locally, which then acts at a long range; and Hh itself diffuses over several cell diameters and acts as a morphogen (3-5). Unlike Drosophila, which has one member of the Hh family, mice have three - Sonic hedgehog (Shh), Indian hedgehog (Ihh), and Desert hedgehog (Dhh) - with Shh being the best studied (Table 1). Shh is expressed at the ventral end of the neural tube (floor plate) and underlying notochord, and patterns the neural tube along its dorsoventral axis (6). Several pieces of evidence support the notion that this patterning is mediated by a direct morphogen activity of Shh. In vitro assays using undifferentiated neural tube explants demonstrate that different dosages of Shh can induce

\footnotetext{
Address correspondence to: Andrew P. McMahon, Department of Molecular and Cellular Biology, The Biolabs, Harvard University, 16 Divinity Avenue, Cambridge, Massachusetts 02138 , USA. Phone: (617) 496-3757; Fax: (617) 496-3763;

E-mail: amcmahon@mcb.harvard.edu.

Conflict of interest declared: A.P. McMahon is a scientific advisor to Curis.

Nonstandard abbreviations used: Hedgehog (Hh); Sonic hedgehog (Shh); Indian hedgehog (Ihh); Desert hedgehog (Dhh); zone of polarizing activity (ZPA); Patched (Ptc); Smoothened (Smo); Dispatched (Disp); sterol-sensing domain (SSD); glycosylphosphatidylinositol (GPI); Tout velu (Ttv); heparan sulfate proteoglycan (HSPG); Hedgehog-interacting protein (Hip).
}

different cell types, where the relative dosages required to induce certain cell fates are consistent with their positions within the concentration gradient in vivo (7, 8). More recently, it has become possible to visualize the endogenous Shh gradient covering the ventral half of the neural tube by immunofluorescence and immunohistochemistry $(9,10)$. In addition, the cellautonomous activation or inactivation of the pathway using mutant forms of receptor components results in autonomous changes of cell fates, confirming the direct role of Hh signaling $(11,12)$. The limb is another place where Shh may function as a morphogen. Shh is expressed in the posterior distal mesenchyme of the limb bud, called the zone of polarizing activity (ZPA), and makes a long-range gradient along the anteroposterior axis $(13,14)$. This concentration gradient is believed to be important in specifying digit identities across the limb bud, with high dosages of Shh close to the ZPA inducing posterior digits and low dosages inducing anterior digits $(13,15,16)$. In addition to its importance during development, inappropriate activation of the Hh pathway has been implicated in human tumors such as basal cell carcinoma, medulloblastoma, fibrosarcoma, and rhabdomyosarcoma (17).

Not surprisingly, significant effort has been devoted to uncovering the molecular mechanism of this pathway (1). Genetic and biochemical studies have established the current model in which $\mathrm{Hh}$ receptor Patched (Ptc) is a negative regulator of the pathway repressing the downstream activator Smoothened (Smo), and binding of $\mathrm{Hh}$ to Ptc abrogates this inhibition, leading to cellular responses via specific transcription factors known in the fly as $\mathrm{Ci}$ and in the mouse as Gli. During the past decade, much excitement has been generated by the discovery of the unusual posttranslational modifications that the Hh protein undergoes (18): the addition of cholesterol (19), which is unprecedented, and palmitoylation (20), which is normally found in cytoplasmic proteins. Here, we will review our current understanding of the mechanism and biological relevance of the cholesterol modification of $\mathrm{Hh}$, with additional discussion of the role of palmitoylation, which has come to light more recently.

Mechanistic basis of cholesterol modification of $\mathrm{Hh}$ $\mathrm{Hh}$ is produced as an approximately $46-\mathrm{kDa}$ precursor. In addition to the removal of the signal peptide, it is 
Table 1

Mammalian Hh genes: some of their normal roles and pathological associations

\begin{tabular}{|c|c|c|}
\hline Hh family member & Role & Human pathology of Hh pathway disruption \\
\hline \multirow[t]{3}{*}{ Sonic hedgehog (Shh) } & Cell proliferation (including neural tissue, hair, tooth, whisker, gut) & Skin and CNS tumors \\
\hline & Cell fate specification (including neural tissues, limb, somites) & Holoprosencephaly and digit anomalies \\
\hline & Morphogenesis of organs (including lung branching, tooth, hair, prostate) & \\
\hline \multirow[t]{3}{*}{ Indian hedgehog (Ihh) } & Cell proliferation (cartilage, gut) & Abnormal skeletal development \\
\hline & Bone development & \\
\hline & Endothelial cell induction & \\
\hline Desert hedgehog (Dhh) & Gonad and peripheral nerve organization & Infertility and disorganization of nerve sheath \\
\hline
\end{tabular}

For comprehensive reviews and references see refs. 1 and 57.

further processed by internal cleavage to generate a 19$\mathrm{kDa}$-terminal fragment $(\mathrm{N}-\mathrm{Hh})$ and a $25-\mathrm{kDa}$-terminal fragment $(\mathrm{C}-\mathrm{Hh})$ with the concomitant addition of cholesterol at the C-terminus of $\mathrm{N}-\mathrm{Hh}\left(\mathrm{N}-\mathrm{Hh}^{\text {chol }}\right)$ $(19,21,22)$. N-Hh has all the known signaling activity of $\mathrm{Hh}$, while $\mathrm{C}-\mathrm{Hh}$ is responsible for this processing reaction $(7,8,13,21,23)$.

The fact that purified Hh protein from a bacterial source can undergo cleavage in vitro first indicated that this is an autoproteolytic process (21), and the concentration-independent kinetics of the reaction further suggested that it occurs by an intramolecular mechanism (23). Based on the analysis of different forms of mutant $\mathrm{Hh}$ proteins, $\mathrm{C}-\mathrm{Hh}$ was found to be the catalytic domain, whereas most of $\mathrm{N}-\mathrm{Hh}$ is dispensable (21, 23). The active site residues in $\mathrm{C}-\mathrm{Hh}$ have been identified from its crystal structure and confirmed by sitedirected mutagenesis (24). Further biochemical analysis led to the following model of a two-step reaction (Figure 1). First, the thiol group of the cysteine at the cleavage site makes a nucleophilic attack on the carbonyl group of the preceding residue, glycine, resulting in a thioester intermediate. Second, cholesterol, most likely by its $3-\beta$ hydroxyl group, attacks this thioester to form an ester-linked adduct to N-Hh and free C-Hh (19, 25 ). While the second step can be driven by other nucleophiles in cell-free systems, there seems to be a specific requirement for sterol in vivo, since sterol deprivation blocks autoproteolysis of $\mathrm{Hh}$ in cultured cells (26).

Several other proteins are likely to use autoprocessing mechanisms similar to that of Hh proteins. The nematode Caenorhabditis elegans has ten proteins with C-terminal regions homologous to $\mathrm{C}-\mathrm{Hh}$, though no bona fide Hh orthologs have been identified in this species (27). Further, metabolic labeling of COS-7 cells with $\left[{ }^{3} \mathrm{H}\right]$ cholesterol revealed its incorporation into several unidentified proteins, consistent with cholesterol addition to other mammalian proteins (19). However, to date, the Hh family is the only example proven to have cholesterol modification.

Interestingly, C-Hh shows sequence and structural homology to self-splicing proteins (24). Since the first step of the protein self-splicing is also the replacement of a peptide bond with a thioester or ester, this suggests the presence of a common evolutionary precursor with the functional module for the thioester/ester formation. From this ancestor, the two protein families must have diverged such that they can achieve cholesterol modification $(\mathrm{Hh})$ or protein splicing (self-splicing proteins) through subsequent steps.

Hh autoprocessing appears to occur early in the secretory pathway, either within the endoplasmic reticulum or soon after entry into the Golgi apparatus. In vitro translation of $\mathrm{Hh}$ in the presence of microsomes gives rise to the mature protein (21), and the precursor cannot proceed past the cis-Golgi cisterna within a cell, evidenced by its sensitivity to endoH glycosidase (26). This may explain the lack of activity of the uncleavable mutant forms of Hh in fly embryos despite their intact signaling domains, for without processing, these proteins are not expected to be secreted from cells $(21,23)$.

\section{Developmental consequences of cholesterol modification of $\mathrm{Hh}$}

One outcome of cholesterol modification is the tethering of $\mathrm{N}-\mathrm{Hh}^{\text {chol }}$ to the membrane of producing cells. When expressed in tissue culture, $\mathrm{N}-\mathrm{Hh}^{\text {chol }}$ is barely detectable in the medium, and the vast majority of the protein remains associated with cells $(7,21-23,25,26)$. In contrast, $\mathrm{N}-\mathrm{Hh}^{\text {unmod }}$, a protein engineered by putting a stop codon at the cleavage point so that it cannot receive cholesterol but otherwise has the same sequence of amino acids as $\mathrm{N}-\mathrm{Hh}^{\text {chol }}$, is efficiently secreted from the cultured cells $(7,22,23,25)$. These results seemed incompatible with the proposed long-range morphogen activity of $\mathrm{N}-\mathrm{Hh}^{\text {chol }}$, and they brought into question the in vivo role of this lipid anchor in Hhmediated signaling processes.

The importance of the cholesterol modification in the context of the embryo development has been tested by expressing the unmodified protein. In Drosophila, the expression of N-Hh ${ }^{\text {unmod }}$ in the normal Hh expression domain results in a gain-of-function phenotype with an abnormally wide range of signaling $(25,28)$. Thus, cholesterol appears to be required to limit diffusion of $\mathrm{N}-\mathrm{Hh}^{\text {chol, }}$, a result consistent with the above-mentioned cell culture experiments. Surprisingly, a similar experiment in mice led to the opposite conclusion: Mouse embryos with $\mathrm{N}$-Shh unmod in place of the wild-type protein show limb patterning defects where the posterior-most digits (digits 5 and 4) that are specified by high doses of Shh close to the ZPA are normal, but anterior digits (digits 3 and 2), which form at a distance from the 
ZPA, are absent (13). This result, together with the posteriorly restricted expression pattern of Shh target genes, suggests that cholesterol modification is essential for the long-range activity of Shh. The discrepancy between the fly and mouse data might reflect differences between the tissues studied in each case. In the fly ectoderm and wing disc, Hh signals within a continuous sheet of epithelium, while in the mouse limb bud, the signaling occurs across the mesenchyme. The area of the gradient formed in mice is also much larger. In addition, the different methods used to express $\mathrm{N}-\mathrm{Hh}^{\text {unmod }}$ or N-Shh ${ }^{\text {unmod, }}$ namely, overexpression using the UAS/GAL4 system in flies versus expression from the endogenous $S h b$ locus in mice, may explain the contrasting results. However, in both systems, it is clear that the cholesterol modification of $\mathrm{Hh}$ is required to confer the correct range and shape to the morphogen gradient and that cholesterol is not absolutely necessary for the signaling activity of the protein.

\section{Cholesterol and the morphogen gradient}

How $\mathrm{N}-\mathrm{Hh}^{\text {chol }}$ is released from producing cells to exert long-range activity is not fully understood, but genetic screens in Drosophila have identified one component dedicated to this process, Dispatched (Disp) (28). Disp is a putative 12-pass transmembrane protein with a sterolsensing domain (SSD). This domain, first identified in proteins implicated in cholesterol homeostasis or trafficking, is required for the sterol-dependent regulation of these activities. The presence of this domain in proteins associated with $\mathrm{Hh}$ release and reception $(\mathrm{Ptc})$ is intriguing, but the role of their SSDs is unclear. In Disp mutants, $\mathrm{N}-\mathrm{Hh}^{\text {chol }}$ is not released to the target field but instead accumulates in the signal-producing cells. Importantly, $\mathrm{N}-\mathrm{Hh}^{\text {unmod }}$ does not require Disp for secretion, and $\mathrm{Hh}$ with noncholesterol anchors, such as a transmembrane domain $\left(\mathrm{N}-\mathrm{Hh}^{\mathrm{TM}}\right)$ or glycosylphosphatidylinositol (N-Hh $\left.{ }^{\mathrm{GPI}}\right)$, can signal only to immediately adjacent cells, even in the presence of Disp. Possible functions of Disp include roles in the intracellular trafficking of $\mathrm{N}-\mathrm{Hh}^{\text {chol }}$ in the secretory pathway, or displacement of the cholesterol anchor from the membrane once it reaches the cell surface. The fact that $\mathrm{N}-\mathrm{Hh}^{\text {chol }}$ can signal within its expression domain in Disp mutants implies that at least some ligand can get to the cell surface and thus favors the second model (29). Recently, $\mathrm{N}$-Shh ${ }^{\text {chol }}$ was shown to form hexamers in solution, presumably with the lipid modifications buried inside (14). In light of this finding, Disp might be involved in the formation of a signaling aggregate that is then capable of diffusing away from Hh-producing cells.

Simply liberating Hh from producing cells does not suffice to make a morphogen gradient, however. $\mathrm{N}-\mathrm{Hh}^{\text {chol, }}$, but not N-Hh${ }^{\text {unmod, }}$, requires the activity of tout velu (ttv) in the target cells to move across this field (30). Ttv is an enzyme involved in heparan sulfate proteoglycan (HSPG) biosynthesis, so the interaction of $\mathrm{N}-\mathrm{Hh}^{\text {chol }}$ with HSPG may be important for its diffusion (31). Ptc also plays an important role in shaping the gradient. This receptor not only transduces the $\mathrm{Hh}$ signal but also binds and internalizes $\mathrm{Hh}$ to restrict the range of diffusion $(9,32)$. In the absence of Ptc, $\mathrm{N}-\mathrm{Hh}^{\text {chol }}$ shows unrestrained movement, much like $\mathrm{N}-\mathrm{Hh}^{\text {unmod }}$. Conversely, effective sequestration by Ptc requires the cholesterol moiety $(13,28)$. The SSD in Ptc was initially thought to enhance the interaction between $\mathrm{N}-\mathrm{Hh}^{\text {chol }}$ and Ptc by binding cholesterol directly, but this prediction is difficult to reconcile with the finding that $\mathrm{N}-\mathrm{Shh}^{\text {unmod }}$ and $\mathrm{N}-\mathrm{Shh}^{\text {chol have }}$ similar binding affinities for Ptc (20). More importantly, mutations in the SSD of Ptc do not affect sequestration of $\mathrm{Hh}$, although in some cases they yield dominant negative alleles that are unable to repress Hh signaling through Smo (33-35). Therefore, the enhanced sequestration of $\mathrm{N}-\mathrm{Hh}^{\text {chol }}$ by Ptc is most likely due to some other cholesterol-dependent process, such as lipid raft association (Simons, this Perspective series, ref. 36; also see below). ptc is itself a transcriptional target of the Hh pathway, providing a feedback mechanism through which Hh controls its own gradient. In vertebrates, the gradient is further refined by Hedgehog-interacting protein (Hip), another antagonist of Hh signaling (37). Hip also appears to function by sequestering Hh ligand. Because Hip does not have an SSD, it will be interesting to see whether it antagonizes the cholesterol-modified and unmodified proteins with the same efficiency.

In summary, the current model for the formation of Hh concentration gradient suggests a process far different from simple diffusion (Figure 2). Cholesterolmodified Hh proteins spread into the target field through multiple interactions with other components of the pathway. These interactions in turn allow layers of regulation, which might be important to make the precise and rigid morphogen gradient appropriate to

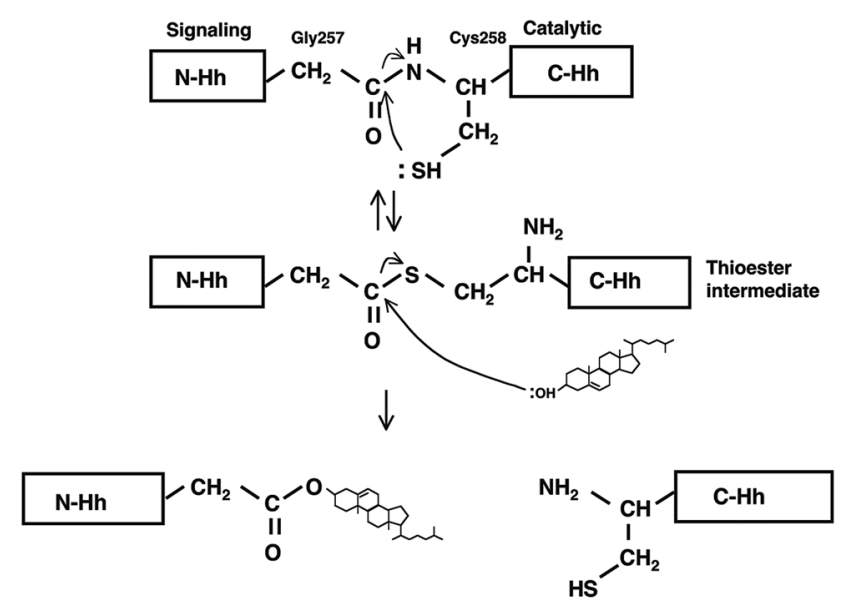

Figure 1

The mechanism of Hh autoprocessing. In Drosophila Hh, the cleavage takes place between Gly257 and Cys258. In the first step, the thiol group of Cys258 makes a nucleophilic attack on the carbonyl group of Gly257 to replace the peptide bond with a thioester. Subsequently, cholesterol attacks the same carbon in the thioester intermediate, which results in the covalent attachment of cholesterol to $\mathrm{N}-\mathrm{Hh}$ and release of $\mathrm{C}-\mathrm{Hh}$. Both steps of the reaction depend on the catalytic activity of $\mathrm{C}-\mathrm{Hh}$, while the signaling activity resides in the $\mathrm{N}$-terminal peptide. Adapted from ref. 49. 


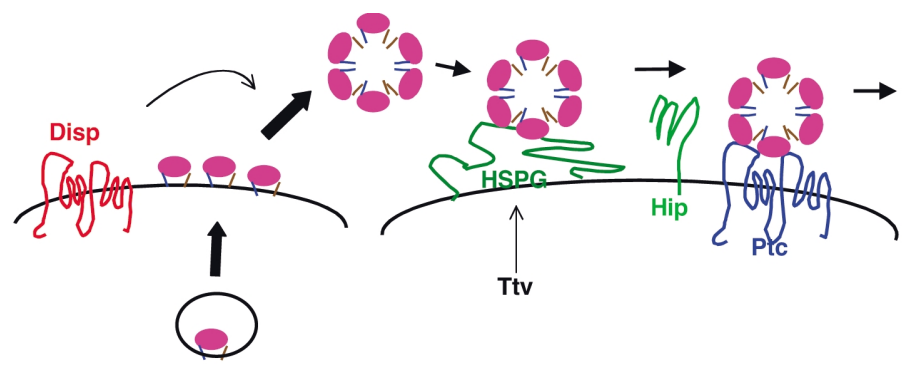

Figure 2

Release and movement of lipid-modified Hh. Secretion of $\mathrm{N}-\mathrm{Hh}^{\text {chol }}$ (with palmitoylation) requires the action of Disp, possibly to override the affinity of the lipid anchors to the membrane. Hexamerization of the ligand may facilitate this process by hiding the lipids inside the complex. Once released, $\mathrm{N}-\mathrm{Hh}^{\text {chol }}$ moves from one cell to another in a process dependent on HSPG whose synthesis requires Ttv. Ptc and Hip limit Hh diffusion by sequestering the ligand.

each situation. For example, dynamic expression of EXTs (mammalian homologs of Ttv) during mouse development might modulate the Shh gradient in a tissue- and stage-specific manner.

\section{Cholesterol as a lipid raft targeting signal}

It is likely that the role of cholesterol modification extends beyond its effect on protein diffusion. One possible function of cholesterol is in targeting of a protein to lipid rafts, as demonstrated by the association of N-Hhchol with the rafts (38). Lipid rafts are sphingolipid- and cholesterol-rich microdomains in lipid bilayers, which associate with specific proteins and behave as assemblies (39). They are thought to be important in some signaling pathways by acting as organizing centers to concentrate the signaling components, and to be important in protein sorting within an epithelium. Concentration of N-Shh chol in lipid rafts may promote its hexamerization, or the interaction with Ptc, which also accumulates in these membrane microdomains (40). Support for a role in protein sorting is hinted at by the subcellular localizations of Hh proteins in Drosophila ectoderm, where $\mathrm{N}-\mathrm{Hh}^{\text {chol }}$ appears as punctate basolateral structures while $\mathrm{N}-\mathrm{Hh}^{\text {unmod }}$ shows diffuse apical localization (25). However, the functional significance of this distribution is unknown.

\section{Palmitoylation of $\mathrm{Hh}$}

While N-Shh ${ }^{\text {chol }}$ has been reported to have higher activ-

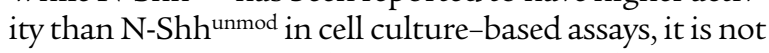
clear whether this is a direct effect, or an indirect effect due to the possible facilitation of the second modification, palmitoylation $(14,20)$. Palmitoylation occurs at the N-terminus of the mature signaling domain and enhances its activity (41). The molecular basis of this increased potency is unclear, because it is not accompanied by an increase in binding affinity to Ptc. As the hydrophobicity of the moiety appears to be more important than its specific chemical nature, increased membrane association might be involved. In Drosophila, two lines of evidence suggest that the palmitoylation of $\mathrm{Hh}$ is absolutely required for its activity. First, a single gene variably named sightless (sit), skinny hedgehog (ski), central missing ( $\mathrm{cmn}$ ), and rasp encodes a putative membranebound acyl transferase required for the palmitoylation of Hh $(29,42-44)$. In the mutants of this gene, Hh proteins are inactive regardless of their cholesterol modifi- cations. Second, a form of Hh that cannot be palmitoylated because of a point mutation at its modification site lacks activity, and even displays dominant negative effect when overexpressed in the presence of normally processed Hh proteins $(43,45)$. For Shh, the equivalent mutants retain activity to varying degrees depending on the assays, implying species- and tissue-specific effects of palmitoylation $(45,46)$. Whether cholesterol enhances the potency of the ligand independently of palmitoylation could be determined by comparing the activities of N-Shh unmod and N-Shh ${ }^{\text {chol }}$ where the palmitoylation site has been modified.

In addition to increasing the specific activity, palmitoyl groups may cooperate with cholesterol to anchor Hh proteins on the membrane, or to target them to lipid rafts. Indeed, indirect evidence from cell culture studies indicates that Shh proteins that are both palmitoylated and cholesterol-modified bind to the membrane more tightly than those with only a cholesterol linkage (20). Also, double acylation is required to direct several other proteins to lipid rafts while single acylation is not sufficient (39). By analogy, both a cholesterol and a palmitoyl group might be necessary for incorporation of $\mathrm{Hh}$ proteins into lipid rafts.

\section{Comparison of cholesterol and other lipid modifications}

Lipid modification is a common strategy for targeting a protein to the membrane, but in most cases (with the exception of GPI anchors), it occurs in cytoplasmic proteins or cytosolic domains of transmembrane proteins (47). GPI and the cholesterol modification of $\mathrm{Hh}$ are similar in that both are attached to extracellular proteins and serve as lipid raft targeting signals. Nevertheless, the finding that $\mathrm{N}-\mathrm{Hh}^{\mathrm{GPI}}$ lacks the longrange activity of $\mathrm{N}-\mathrm{Hh}^{\text {chol }}(28)$ shows that GPI cannot replace cholesterol in $\mathrm{Hh}$. This result is somewhat puzzling, because a recent study has indicated that GPI-linked proteins as well as cytoplasmic proteins anchored to the inner leaflet of the membrane can spread through the epithelium of the fly imaginal disc in membrane vesicles named argosomes, suggesting that the ability to support long-range movement is not limited to cholesterol (48). Either N-Hh ${ }^{\mathrm{GPI}}$ is incorporated into the argosomes inefficiently, or, more likely, the long-range activity of Hh depends on other mechanisms that operate only on cholesterol-modified $\mathrm{Hh}$ and involve Disp and HSPG. 


\section{Cholesterol and Smo activity in the Hh pathway}

Exposure of mammalian embryos to the distal inhibitors of cholesterol biosynthesis or steroidal alkaloids such as jervine and cyclopamine causes holoprosencephaly, the phenotype found in mice and humans with mutations in Shb (49-51). Instead of affecting cholesterol modification of Shh, these steroidal alkaloids appear to inhibit the response in signal-receiving cells $(52,53)$. Further analysis of cyclopamine's effect points to a role in antagonizing Smo activity, possibly directly (54). Although the mechanism by which Smo activity is regulated remains unclear, this observation reveals an additional link between cholesterol and the Hh pathway.

\section{Concluding comments}

Owing to rapid progress over the last several years, we have a good understanding of the mechanism by which Hh becomes cholesterol-modified, but the functional significance of this event is poorly defined. Although in vivo studies with proteins that lack cholesterol demonstrate the importance of this modification in the regulated diffusion of ligand, it is unclear why cholesterol, instead of more common lipid adducts, is used for this purpose. The answer to this question most likely lies in the interaction of the ligand with SSD-containing components of the pathway, Disp and Ptc in the case of Hh. Perhaps the cholesterol-modified ligand and SSD-containing proteins coevolved as a morphogen gradient-generating system. In this regard, it will be informative to identify other cholesterol-modified proteins and see whether they also have cognate SSD proteins or make morphogen gradients. A cholesterol anchor would be just one of many different strategies embryos employ to control protein diffusion, for other morphogens form stable gradients without the use of lipid modifications $(55,56)$. However, the membrane association that results from this moiety might be a particularly effective mechanism for long-range signaling, concentrating ligand in the membrane, and enhancing the probability of receptor-ligand interaction at low concentration thresholds, some distance from the signaling center.

The second link between cholesterol and the Hh pathway - Smo inhibition by steroidal alkaloids underscores another long-standing question with respect to this pathway, namely, the mechanism by which Ptc and Smo activities are regulated in the signal-receiving cells. A growing body of evidence suggests that vesicular trafficking is involved (1), but how cholesterol might fit into this picture remains to be seen. In conclusion, investigations into the role of cholesterol modification have enhanced our understanding of Hh signaling, but they have also unearthed many fascinating questions. The next several years should prove to be as exciting a time in this field as the past few years have been.

\section{Acknowledgments}

We thank Thomas Carroll, Toyoaki Tenzen, and Jing Yu for critical readings of the manuscript, and Mark Wijgerde for helpful discussion. Work in A.P. McMahon's laboratory on the role of vertebrate Hh proteins is supported by grants from the NIH (NS-33642 and DK-56246).

1. Ingham, P.W., and McMahon, A.P. 2001. Hedgehog signaling in animal development: paradigms and principles. Genes Dev. 15:3059-3087.

2. Alexandre, C., Lecourtois, M., and Vincent, J.-P. 1999. Wingless and Hedgehog pattern Drosophila denticel belts by regulating the production of short-range signals. Development. 126:5689-5698.

3. Zecca, M., Basler, K., and Struhl, G. 1995. Sequential organizing activities of engrailed, hedgehog, and decapentaplegic in Drosophila wing. Development. 121:2265-2278.

4. Mullor, J., Calleja, M., Capdevila, J., and Guerrero, I. 1997. Hedgehog activity, independently of decapentaplegic, participates in wing disc patterning. Development. 124:1227-1237.

5. Strigini, M., and Cohen, S.M. 1997. A hedgehog activity gradient contributes to AP axial patterning of the Drosophila wing. Development. 124:4697-4705.

6. Jessell, T.M. 2000. Neuronal specification in the spinal cord: inductive signals and transcriptional codes. Nat. Rev. Genet. 1:20-29.

7. Roelink, H., et al. 1995. Floor plate and motor neuron induction by different concentrations of the amino terminal cleavage product of Sonic hedgehog autoproteolysis. Cell. 81:445-455.

8. Briscoe, J., Pierani, A., Jessell, T.M., and Ericson, J. 2000. A homeodomain protein code specifies progenitor cell identity and neuronal fate in the ventral neural tube. Cell. 101:435-445.

9. Incardona, J., et al. 2000. Receptor-mediated endocytosis of soluble and membrane-tethered Sonic hedgehog by patched-1. Proc. Natl. Acad. Sci. USA. 97:12044-12049.

10. Gritli-Linde, A., Lewis, P., McMahon, A., and Linde, A. 2001. The whereabouts of a morphogen: direct evidence for short- and graded long-range activity of hedgehog signaling peptides. Dev. Biol. 236:364-386.

11. Hynes, M., et al. 2000. The seven-transmembrane receptor smoothened cell autonomously induces multiple ventral cell types. Nat. Neurosci. 3:41-46.

12. Briscoe, J., Chen, Y., Jessell, T., and Struhl, G. 2001. A Hedgehog-insensitive form of Patched provides evidence for direct long-range morphogen activity of Sonic hedgehog in the neural tube. Mol. Cell. 7:1279-1291.

13. Lewis, P., et al. 2001. Cholesterol modification of sonic hedgehog is required for long-range signaling activity and effective modulation of signaling by Ptc1. Cell. 105:599-612.

14. Zeng, X., et al. 2001. A freely diffusible form of Sonic hedgehog mediates long-range signalling. Nature. 411:716-720.

15. Riddle, R.D., Johnson, R.L., Laufer, E., and Tabin, C. 1993. Sonic hedgehog mediates the polarizing activity of the ZPA. Cell. 75:1401-1416.

16. Yang, Y., et al. 1997. Relationship between dose, distance, and time in Sonic Hedgehog-mediated regulation of anteroposterior polarity in the chick limb. Development. 124:4393-4404.

17. Taipale, J., and Beachy, P. 2001. The Hedgehog and Wnt signaling pathways in cancer. Nature. 411:349-354.

18. Ingham, P.W. 2001. Hedgehog signaling: a tale of two lipids. Science. 294:1879-1881.

19. Porter, J.A., Young, K.E., and Beachy, P. 1996. Cholesterol modification of hedgehog signaling proteins in animal development. Science. 274:255-259.

20. Pepinsky, R.B., et al. 1998. Identification of a palmitic acid-modified form of human Sonic hedgehog. J. Biol. Chem. 273:14037-14045.

21. Lee, J.J., et al. 1994. Autoproteolysis in hedgehog protein biogenesis. Science. 266:1528-1537.

22. Bumcrot, D.A., Takata, R., and McMahon, A.P. 1995. Proteolytic processing yields two secreted forms of Sonic hedgehog. Mol. Cell. Biol. 15:2294-2303.

23. Porter, J.A., et al. 1995. The product of hedgehog autoproteolytic cleavage active in local and long-range signalling. Nature. 374:363-366.

24. Hall, T.M.T., et al. 1997. Crystal structure of a Hedgehog autoprocessing domain: homology between hedgehog and self-splicing proteins. Cell. 91:85-97.

25. Porter, J.A., et al. 1996. Hedgehog patterning activity: role of a lipophilic modification mediated by the carboxy-terminal autoprocessing domain. Cell. 86:21-34.

26. Guy, R.K. 2000. Inhibition of sonic hedgehog autoprocessing in cultured mammalian cells by sterol deprivation. Proc. Natl. Acad. Sci. USA. 97:7307-7312.

27. Aspock, G., Kagoshima, H., Niklaus, G., and Burglin, T.R. 1999. Caenorhabditis elegans has scores of hedgehog-related genes: sequence and expression analysis. Genome Res. 9:909-923.

28. Burke, R., et al. 1999. Dispatched, a novel sterol-sensing domain protein dedicated to the release of cholesterol-modified Hedgehog from signaling cells. Cell. 99:803-815. 
29. Amanai, K., and Jiang, J. 2001. Distinct roles of Central missing and Dispatched in sending the Hedgehog signal. Development. 128:5119-5127.

30. Bellaiche, Y., The, I., and Perrimon, N. 1998. Tout-velu is a Drosophila homologue of the putative tumour suppressor EXT-1 and is needed for Hh diffusion. Nature. 394:85-88.

31. The, I., Bellaiche, Y., and Perrimon, N. 1999. Hedgehog movement is regulated through tout velu-dependent synthesis of a heparan sulfate proteoglycan. Mol. Cell. 4:633-639.

32. Chen, Y., and Struhl, G. 1996. Dual roles for Patched in sequestering and transducing Hedgehog. Cell. 87:553-563.

33. Martin, V., Carrillo, G., Torroja, C., and Guerrero, I. 2001. The sterolsensing domain of Patched protein seems to control Smoothened activity through Patched vesicular trafficking. Curr. Biol. 11:601-607.

34. Strutt, H., et al. 2001. Mutations in the sterol-sensing domain of Patched suggest a role for vesicular trafficking in Smoothened regulation. Curr. Biol. 11:608-613.

35. Johnson, R.L., Zhou, L., and Bailey, E.C. 2002. Distinct consequences of sterol sensor mutations in Drosophila and mouse patched homologs. Dev. Biol. 242:224-235.

36. Simons, K., and Ehehalt, R. 2002. Cholesterol, lipid rafts, and disease. J. Clin. Invest. 110:597-603. doi:10.1172/JCI200216390.

37. Chuang, P.-T., and McMahon, A.P. 1999. Vertebrate Hedgehog signaling modulated by induction of a Hedgehog-binding protein. Nature. 397:617-621.

38. Rietveld, A., Neutz, S., Simons, K., and Eaton, S. 1999. Association of sterol- and glycosylphosphatidylinositol-linked proteins with Drosophila raft lipid microdomains. J. Biol. Chem. 274:12049-12054.

39. Simons, K., and Ikonen, E. 1997. Functional rafts in cell membranes. Nature. 387:569-572.

40. Karpen, H.E., et al. 2001. The sonic hedgehog receptor patched associates with caveolin-1 in cholesterol-rich microdomains of the plasma membrane. J. Biol. Chem. 276:19503-19511.

41. Taylor, F.R., et al. 2001. Enhanced potency of human Sonic hedgehog by hydrophobic modification. Biochemistry. 40:4359-4371.

42. Lee, D.J., and Treisman, J.E. 2001. Sightless has homology to transmembrane acyltransferases and is required to generate active Hedgehog protein. Curr. Biol. 11:1147-1152.
43. Chamoun, Z., et al. 2001. Skinny Hedgehog, an acyltransgerase required for palmitoylation and activity of the Hedgehog signal. Science. 293:2080-2084

44. Micchelli, C.A., The, I., Selva, E., Mogila, V., and Perrimon, N. 2002. rasp, a putative transmembrane acyltransferase, is required for Hedgehog signaling. Development. 129:843-851.

45. Lee, J.D., et al. 2001. An acylatable residue of Hedgehog is differentially required in Drosophila and mouse limb development. Dev. Biol. 233:122-136

46. Kohtz, J.D., et al. 2001. N-terminal fatty-acylation of sonic hedgehog enhances the induction of rodent ventral forebrain neurons. Development. 128:2351-2363.

47. Casey, P.J. 1995. Protein lipidation in cell signaling. Science. 268:221-225.

48. Greco, V., Hannus, M., and Eaton, S. 2001. Argosomes: a potential vehicle for the spread of morphogens through epithelia. Cell. 106:633-645.

49. Beachy, P.A., et al. 1997. Multiple roles of cholesterol in Hedgehog protein biogenesis and signaling. Cold Spring Harb. Symp. Quant. Biol. 62:191-204

50. Chiang, C., et al. 1996. Cyclopia and defective axial patterning in mice lacking Sonic hedgehog gene function. Nature. 383:407-413.

51. Roessler, E., et al. 1996. Mutations in the human Sonic Hedgehog gene cause holoprosencephaly. Nat. Genet. 14:357-360.

52. Cooper, M.K., Porter, J.A., Young, K.E., and Beachy, P.A. 1997. Teratogenmediated inhibition of target tissue response to $S h b$ signaling. Science. 280:1603-1606.

53. Incardona, J.P., Gaffield, W., Kapur, R.P., and Roelink, H. 1998. The teratogenic Veratrum alkaloid cyclopamine inhibits sonic hedgehog signal transduction. Development. 125:3553-3562.

54. Taipale, J., et al. 2000. Effects of oncogenic mutations in Smoothened and Patched can be reversed by cyclopamine. Nature. 406:1005-1009.

55. Tabata, T. 2001. Genetics of morphogen gradients. Nat. Rev. Genet. 2:620-630.

56. Teleman, A.A., Strigini, M., and Cohen, S.M. 2001. Shaping morphogen gradient. Cell. 105:559-562.

57. McMahon, A.P., Ingham, P.W., and Tabin, C. 2002. The developmental roles and clinical significance of Hedgehog signaling. Curr. Top. Dev. Biol. In press. 